\title{
Numerical simulation and experimentation of endodontic file using Taguchi DoE
}

\author{
Pravin R. Lokhande, Sachin S. Salunkhe* (D), and Sethuraman Balaguru \\ Vel Tech Rangarajan Dr. Sagunthala R\&D Institute of Science and Technology, Chennai 600062, India
}

Received: 7 January 2021 / Accepted: 26 October 2021

\begin{abstract}
The endodontic file is a tapered, needle shape body used for the preparation of curved human root canals. During the preparation, process files get failed due to the locking action offered by the canal wall. The present study aimed to find the fatigue life of endodontic files at $23^{\circ}, 33^{\circ}$ and $43^{\circ}$ root canal curvature angles. Four brands of files were selected for the present study viz. Hyplex CM, Pro-Taper Next, Hero Shaper, Pro-File Vortex. The strain life analysis done using ANSYS showed that the Hyplex CM file gives the highest fatigue life at $23^{\circ}, 33^{\circ}$ and $43^{\circ}$ root canal curvature angles. Therefore, Hyplex CM file was selected for DoE Taguchi Optimization study. Each experimental reading was conducted on X Smart Plus experimental setup under purely rotary and combined reciprocating-rotary motion, at $500 \mathrm{rpm}, 600 \mathrm{rpm}$ and $700 \mathrm{rpm}$ and $23^{\circ}, 33^{\circ}$ and $43^{\circ}$ root canal curvature angles. Hyplex CM file given maximum fatigue life at $23^{\circ}$ root canal curvature angle and $500 \mathrm{rpm}$ speed of rotation. But if file motion is combined rotary-reciprocating, life reduces. The ANNOVA study showed that $P$-value and significance $F$ are very small, which represented that the regression model is effective.
\end{abstract}

Keywords: Endodontic file / fatigue life / root canal / DoE / ANOVA

\section{Introduction}

The endodontic treatment aims to save the tooth which is infected, decayed or highly susceptible to future infection. The endodontic treatment is also known as root canal treatment [1]. The cleaning and shaping of the infected root canal are known as root canal preparation and filling and sealing of the prepared root canal using biocompatible material Gutta-percha is known as root canal obturation, both root canal preparation and obturation comes under endodontic treatment and is known as root canal treatment $[2,3]$. The endodontic files are used to prepare the root canals these files are tapered drill bit-shaped instruments having continuous helical grooves called flutes. Flutes help debris to come out from the infected root canal during the preparation process [4]. The endodontic file rotates in a curved root canal in presence of infected tissue, debris and oral fluids which offers locking pressure to the rotating file. The locking action of the canal on the endodontic file leads to fatigue failure [5]. This fatigue failure can be assessed using cyclic fatigue or torsion fatigue approach. These approaches help to find the life of the endodontic file. The life of the endodontic file helps the Dentist to make use of the file so that failure of the file in the patient jaw can be avoided during canal preparation.

\footnotetext{
* e-mail: drsalunkhesachin@veltech.edu.in
}

The present study is divided into two parts viz. (i) numerical simulation of the endodontic file and (ii) optimization of the endodontic file using Taguchi Design of Experiments (DoE). The study used finite element analysis and cyclic fatigue test for assessment of fatigue life of endodontic files during the root canal preparation process when placed in $23^{\circ}, 33^{\circ}$ and $43^{\circ}$ root canal curvature angles and under purely rotary, combined reciprocating-rotary motions. The Taguchi DoE optimization was used to find optimized root canal angle and speed of rotation giving maximum fatigue life of the file. Regression analysis and ANNOVA were used for statistical analysis of obtained results.

\section{Literature review}

Fatigue is progressive, confined structural destruction caused to Endodontic files subjected to periodic loading. If applied loads are higher than the design load, a microscopic crack begins forming stress concentrators such as slip bands which propagate suddenly to cause fatigue failure. The stress variations in the Ni-Ti rotary instruments were conducted and it failed to evaluate the life of files. The study used the virtual model to estimate the life of the file and found the locations of failure [6]. The finite element method for analysis of cyclic performance Ni-Ti rotary files made up of different materials was 


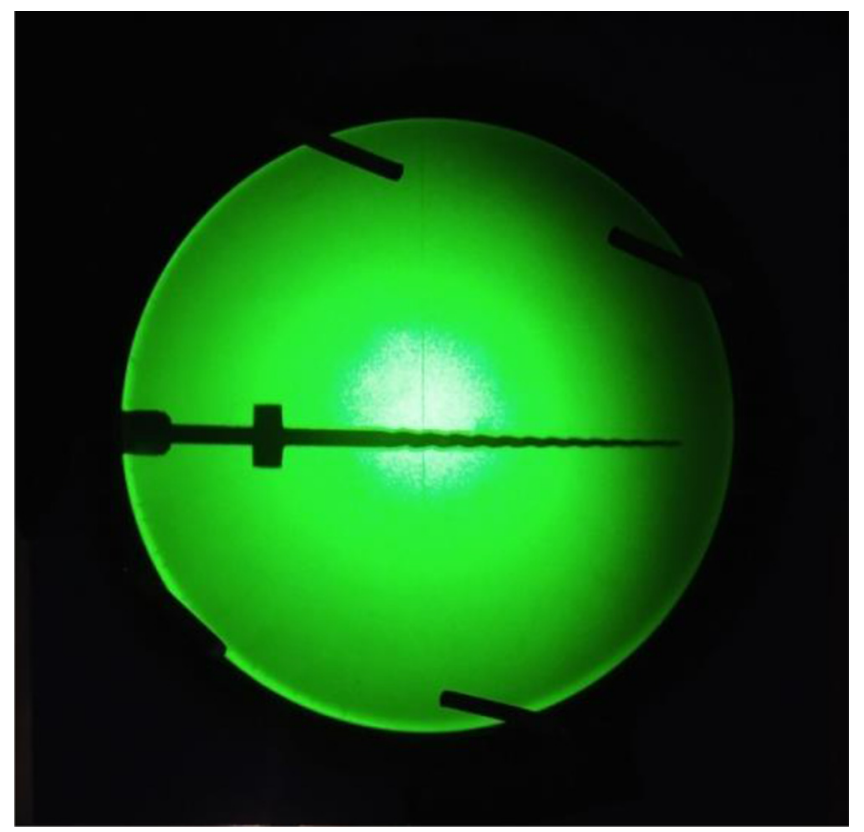

Fig. 1. Endodontic file profile view obtained on optical profile meter.

studied [7]. There is a need to measure the resistance offered by the file to avoid fractures [8]. The instrument fractures are dependent of root canal curvature angle, operating speed, torsion, compression and tension, Endodontic file properties, instrument manufacturing methods/defects, instruments geometry and material fatigue [9]. The stress state for the Endodontic file instrument is affected by many parameters viz. size, taper, crosssectional area, rigidity, flexibility, the direction of rotation of files and forces acting on the rotary instruments during working [10]. The study reported that different methodologies affected the fatigue life of the instruments and therefore the clinical outcome. It is suggested to carry the international standard for similarity in methodology and comparable results [11].

\section{Materials and method}

This section describes two methods viz. (i) Numerical simulation of the endodontic file and (ii) Optimization of the endodontic file using Taguchi Design of Experiments (DoE). The numerical simulation of the endodontic file helped to find out the fatigue life of endodontic files of four brands viz. Hyplex CM, Pro-Taper Next, Hero Shaper, Pro-File Vortex. Out of these four brands of files, the file with the highest fatigue life (best file) was selected for DoE optimization. Taguchi DoE has given the best parameter combination that suits maximum fatigue life.

\subsection{Numerical simulation of endodontic file}

The cyclic fatigue life assessments of Endodontic files have been carried out using finite element analysis for rotary motion at $23^{\circ}, 33^{\circ}$ and $43^{\circ}$ root canal curvature angles. The Endodontic file model of each brand viz. Hyplex CM,

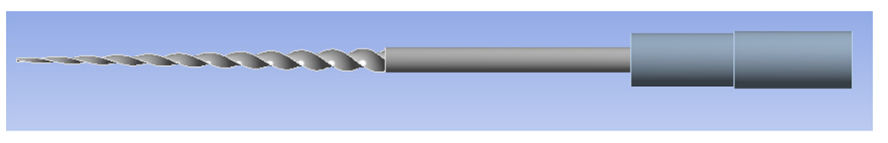

Front view

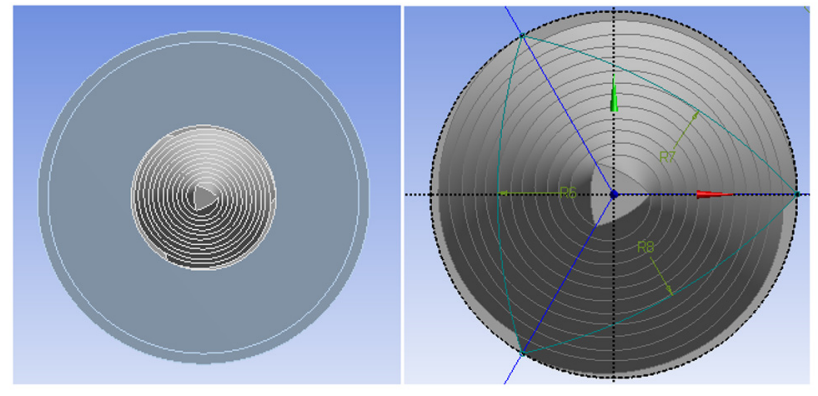

Side view

Fig. 2. CAD model of hyplex CM endodontic file.

Pro-Taper Next, Hero Shaper, Pro-File Vortex were imported to ANSYS to find out the cyclic fatigue by varying root canal curvature angles. ANSYS is the FEA-based analysis software that calculated the fatigue life of endodontic files using the strain-life fatigue analysis approach. In any patient, the root canal angle varies from $0^{\circ}$ to $45^{\circ}$. The present study considered $23^{\circ}, 33^{\circ}$ and $43^{\circ}$ root canal curvature angles.

\subsubsection{Measurement of dimensions and modeling endodontic file}

Profilometry extracts the topological data from the surface of the component. The main aim of profilometry is to obtain surface morphology, length, breadth, depth, crest, trough, pitch, orientation angle. The view of the endodontic file on the optical profile-meter is shown in Figure 1.

The CAD models of Hyplex CM endodontic file was needed to perform fatigue life assessment in ANSYS. The dimensions of the file were measured using optical profilemeter was used to model the endodontic file (shown in Fig. 1). These CAD models of file were used for assessment of fatigue life in ANSYS shown in Figure 2.

\subsubsection{Material property inputs and meshing of endodontic file}

In order to give the input of material properties of nickeltitanium alloy to ANSYS software, various tests need to be performed to obtain the properties of the material. The past studies have been already investigated these material properties and used them for their study [5]. The material properties are shown in Tables 1 and 2 .

Any continuous object has infinite degrees of freedom and it's not just possible to solve the problem in this format. Finite element analysis reduces the degree of freedom from infinite to finite with the help of discretization or meshing. The meshing divided the endodontic file CAD model into 47230 nodes and 13118 elements. Meshing 
Table 1. Material property for nickel-titanium alloy.

\begin{tabular}{llll}
\hline Sr. No & Material property & Numeric value & Unit \\
\hline 01 & Density & 1500 & $\mathrm{~kg} . \mathrm{m}^{-3}$ \\
02 & Young's modulus & 60000 & $\mathrm{MPa}$ \\
03 & Poisson's ratio & 0.33 & - \\
04 & Bulk modulus & $2.2556 \mathrm{E}+10$ & $\mathrm{~Pa}$ \\
05 & Shear modulus & $2.2556 \mathrm{E}+10$ & $\mathrm{~Pa}$ \\
\hline
\end{tabular}

Table 2. Linear elastic properties for nickel-titanium alloy.

\begin{tabular}{llll}
\hline Sr. No & Material property & Numeric value & Unit \\
\hline 01 & Sigma SAS & 482.6 & $\mathrm{MPa}$ \\
02 & Sigma FAS & 620.5 & $\mathrm{MPa}$ \\
03 & Sigma SSA & 379.2 & $\mathrm{MPa}$ \\
04 & Sigma FSA & 103.4 & $\mathrm{MPa}$ \\
05 & Epsilon & 0.068 & $\mathrm{~mm}^{-1}$ \\
\hline
\end{tabular}

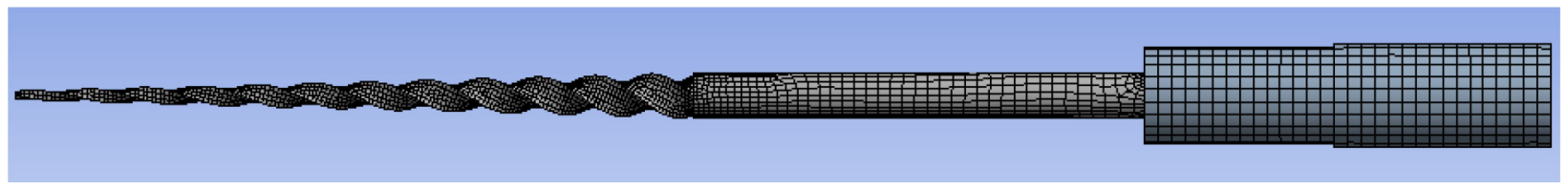

Fig. 3. Meshed model of hyplex CM endodontic file.

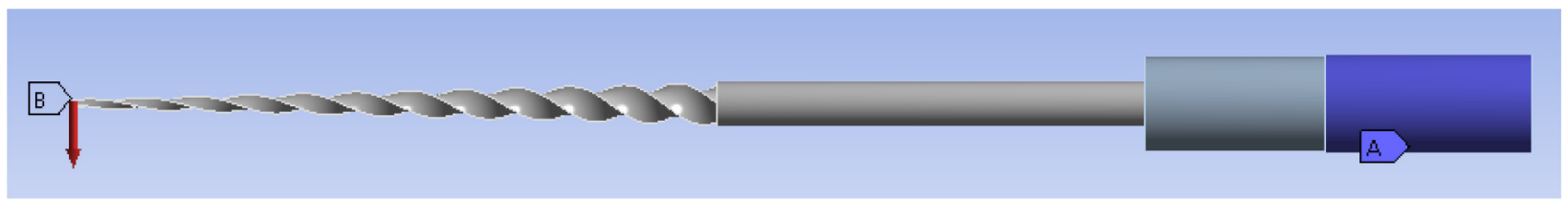

Fig. 4. Boundary conditions input in hyplex CM endodontic file.

used two types of elements viz. 10-noded tetrahedral elements (Solid187) and 20-noded hexahedral elements (Solid186). Looking at the shape of the tetrahedral element, it can be easily adjusted on the curved/complex profile of the endodontic file. Therefore, the tetrahedral element was selected for the meshing body of the endodontic file. On the other hand, the handle is simple in structure and therefore, was meshed using hexahedral elements. The mesh model of Hyplex CM file is shown in Figure 3 .

\subsubsection{Boundary conditions input for endodontic file}

Defining boundary conditions and loading conditions means fixing the value of displacement and load on a specific area of the model. Thus, the model correctly constrained can give highly accurate results. The endodontic file was fixed at the handle end and a force of $0.24 \mathrm{~N}$ was applied to cause deflection of the tip by $1.88 \mathrm{~mm}$ (shown in Fig. 4). When the endodontic file was inserted into $43^{\circ}$ root canal its tip gets deflected by $1.88 \mathrm{~mm}$ from the axis line. For simulation of this deflection in ANSYS 0.24 N force was applied to cause $1.88 \mathrm{~mm}$ deflection which constitutes $43^{\circ}$ root canal angle. The experimental study used an acrylic block in which the root canal cavity was made to simulate the root canal but in ANSYS we avoiding this which saved the time required to build the root canal CAD model.

\subsubsection{Strain life analysis using ANSYS}

The ANSYS is used to perform the strain-life fatigue analysis. Strain-life analysis requires the input of nickeltitanium alloys material properties such as strength coefficient, strength exponent, ductility coefficient, ductility exponent, cyclic strength coefficient and cyclic strain hardening exponent. The strain analysis provides the fatigue life in terms of the number of reversals. The fatigue life of the endodontic file can be calculated using the 


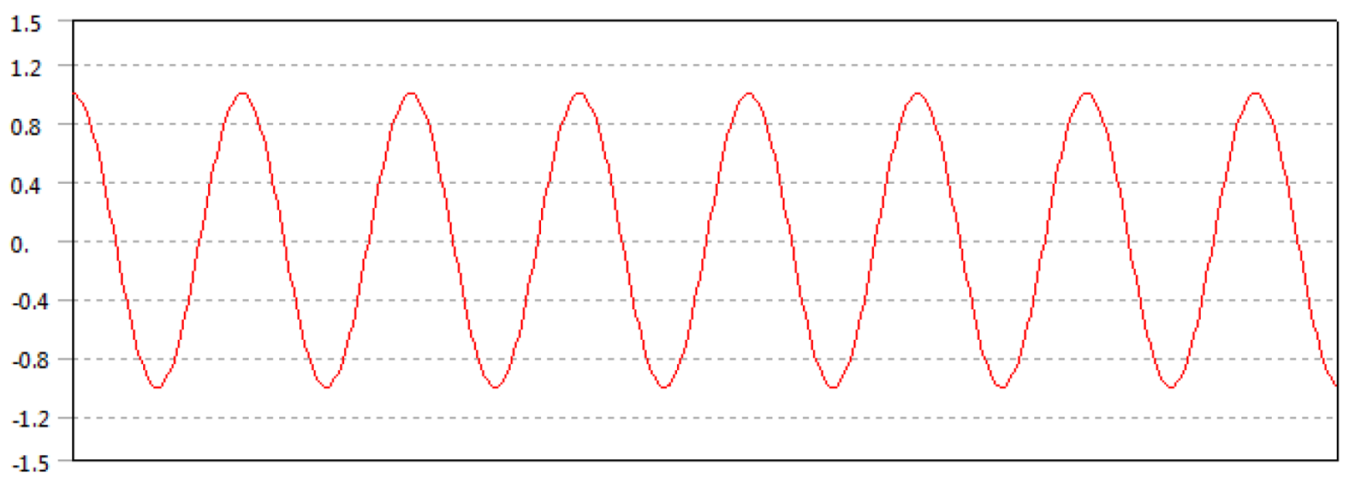

Fig. 5. Reversible loading.

Mean Stress Correction Theory

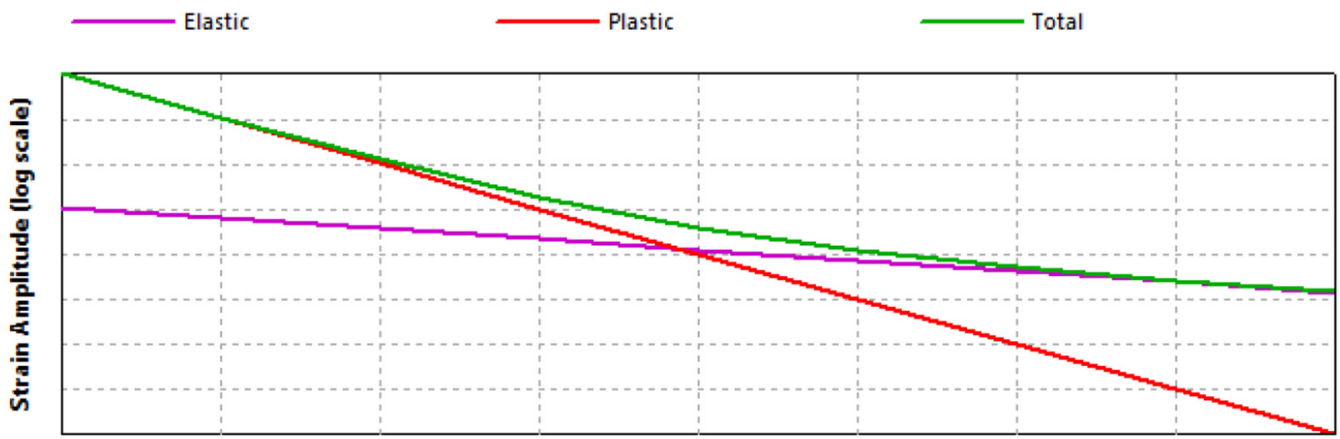

Reversals to Failure, 2N (log scale)

Fig. 6. Strain-life curve.

following equation.

$$
\begin{aligned}
& \text { The cyclic fatigue life of endodontic file } \\
& =\frac{\text { Number of reversals }}{2} \text {. }
\end{aligned}
$$

The geometric nonlinearity considered in the static analysis: Under solver controls 'Large Deflection' option selected. Fully reversed type loading was used as shown in Figure 5. The various analysis parameters inputs for analysis used includes Fatigue Stress Concentration factor $=1$; Type of approach used in fatigue analysis: Strain-life (The strain life curve for analysis is shown in Fig. 6); Stress component considered: Equivalent Stress; No mean stress theory was considered. The other strain life parameters are tabulated in Table 3 .

\subsubsection{Fatigue life of hyplex CM endodontic file based on ANSYS report}

The infinite fatigue life was defined at $10^{7}$ cycles or above. The strain-life approach measures life in terms of reversals. The number of reversals to failure is equal to one-half of the fatigue life of the file. The minimum number of reversals observed for hyplex CM endodontic file is 5055 (shown in Fig. 7). So, the life of Hyplex CM endodontic file in the terms of the number of cycles is 2527 number of rotations.
Similarly, the analysis procedure described for Hyplex CM endodontic file was followed for fatigue analysis of remaining files viz. Pro-Taper Next, Hero Shaper and ProFile Vortex at $23^{\circ}, 33^{\circ}$ and $43^{\circ}$ root canal curvature angle. The results were noted in Table 4 and it is found that Hyplex CM endodontic files show the highest fatigue life at $23^{\circ}, 33^{\circ}$ and $43^{\circ}$ root canal curvature angle. Therefore, Hyplex CM file was selected for DoE Taguchi Optimization study.

\subsection{DoE of the endodontic file using Taguchi method}

The life of the endodontic file gets affected due to root canal preparation process parameters viz. root canal curvature angle, speed of endodontic file and type of endodontic file motion. Therefore, it had been decided to conduct Taguchi DoE optimization to find parameter combinations giving maximum fatigue life. The Hyplex CM nickel-titanium endodontic file of $25 \mathrm{~mm}$ length and $6 \%$ taper was selected. Numerical simulation study resulted that Hyplex CM endodontic files show highest fatigue life at $23^{\circ}, 33^{\circ}$ and $43^{\circ}$ root canal curvature angle. Therefore, Hyplex CM file was selected for DoE Taguchi Optimization study.

This section describes fatigue life assessment using X-Smart Plus and implementation of Taguchi DoE optimization. 
Table 3. Strain life parameters considered for the nickel-titanium file material.

\begin{tabular}{lll}
\hline Strain life parameters & Numeric value & Unit \\
\hline Display curve type & Strain life & - \\
Strength coefficient & 705 & $\mathrm{MPa}$ \\
Strength exponent & -0.06 & - \\
Ductility coefficient & 0.68 & - \\
Ductility exponent & -0.6 & - \\
Cyclic strength coefficient & 733 & $\mathrm{MPa}$ \\
Cyclic strain hardening exponent & 0.1 & - \\
\hline
\end{tabular}

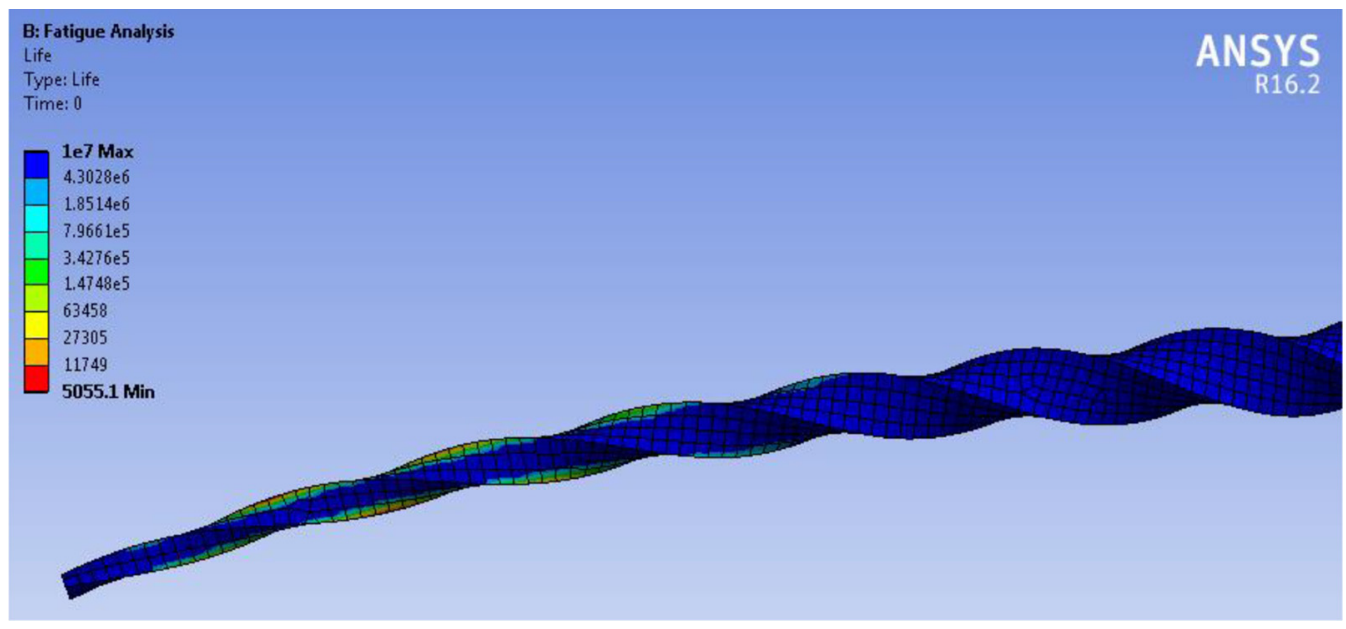

Fig. 7. Fatigue life for hyplex CM endodontic file (isometric view).

Table 4. Effect of rotary motion on fatigue life of endodontic file.

\begin{tabular}{lll}
\hline $\begin{array}{l}\text { Endodontic file } \\
\text { brand/make }\end{array}$ & $\begin{array}{l}\text { Approximate root } \\
\text { canal angulations } \\
\left(\alpha^{\circ}\right)\end{array}$ & $\begin{array}{l}\text { Cyclic fatigue resistance in term of } \\
\text { No. of rotations (n) } \\
\text { Using FEA }\end{array}$ \\
\hline $\begin{array}{l}\text { Hyplex CM } \\
\text { Pro-Taper next }\end{array}$ & & 8112 \\
Hero Shaper & $23^{\circ}$ & 1905 \\
Pro-File Vortex & & 3693 \\
Hyplex & & 6000 \\
Pro-Taper next & $33^{\circ}$ & 4213 \\
Hero Shaper & & 1241 \\
Pro-File Vortex & & 3210 \\
Hyplex & & 3486 \\
Pro-Taper next & $43^{\circ}$ & 2527 \\
Hero Shaper & & 518 \\
Pro-File Vortex & & 1448 \\
\hline
\end{tabular}

\subsubsection{Fatigue life assessment using X-Smart Plus}

The cyclic fatigue life assessments of Endodontic files had been carried out using X Smart Plus experimental setup (shown in Fig. 8) for rotary and combined rotaryreciprocating motions at $23^{\circ}, 33^{\circ}$ and $43^{\circ}$ root canal curvature angles. During each experimental run, the endodontic file was inserted into the artificial root canal cavity in the acrylic block to measure the fatigue life. The nickel-titanium alloy files have made it easy to prepare the root canal by eliminating the difficulties available with stainless-steel files such as canal transportation, ledges, 


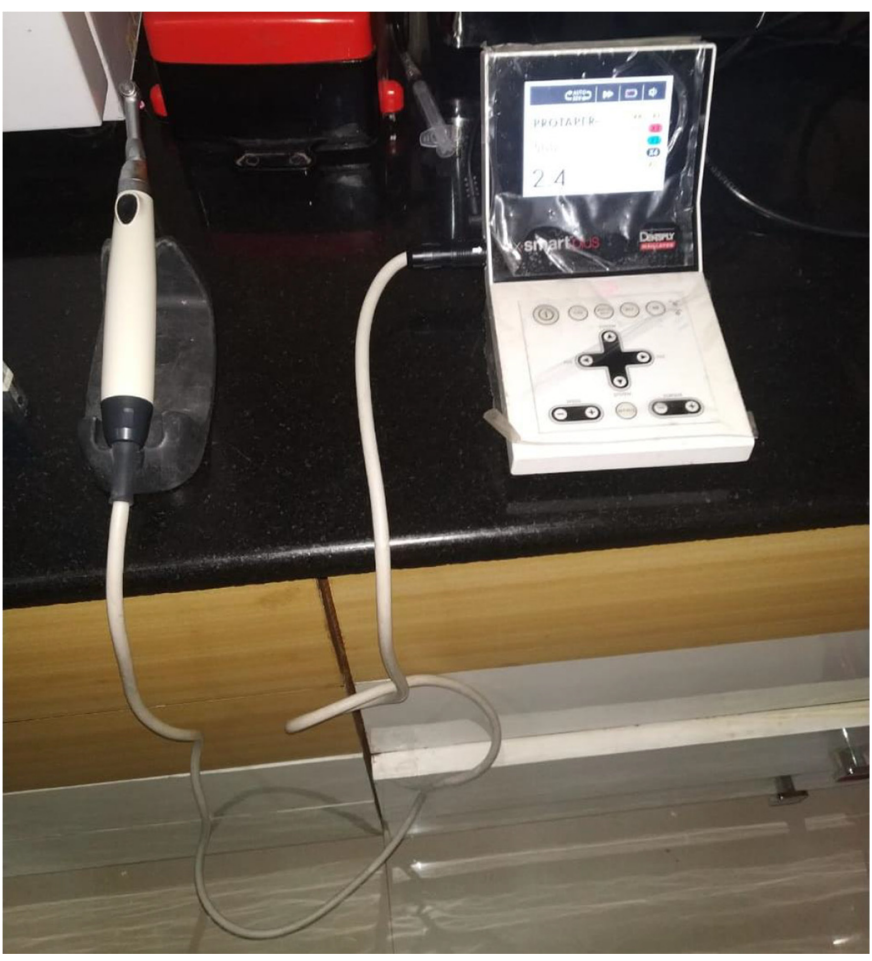

Fig. 8. X Smart Plus experimental setup.

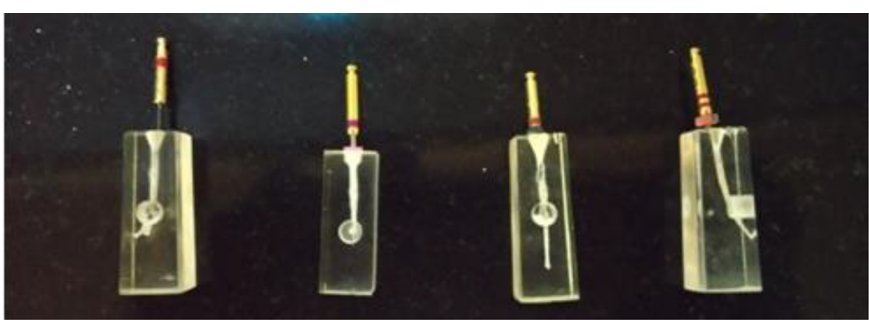

Fig. 9. Fractured samples during root canal preparation process.

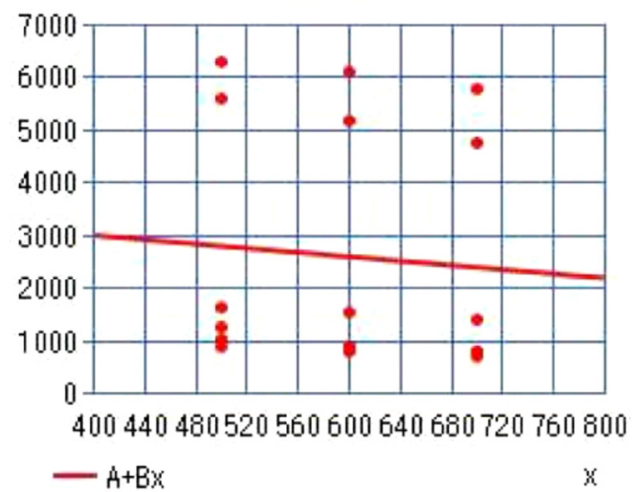

Fig. 10. Simple linear regression curve.

Table 5. Contributing factors and their levels.

\begin{tabular}{llll}
\hline \multirow{2}{*}{ Levels } & \multicolumn{3}{c}{ Root canal preparation parameters } \\
\cline { 2 - 4 } & Motion type & Root canal angle & Speed of file \\
\hline 1 & Purely rotary & $23^{\circ}$ & 500 \\
2 & Combined rotary-reciprocating & $33^{\circ}$ & 600 \\
3 & - & $43^{\circ}$ & 700 \\
\hline
\end{tabular}

zips and perforation [12] (Peters 2004). Therefore, nickeltitanium alloy endodontic files were used in this study. To conform with standard conditions for each experimental run new endodontic file ( $25 \mathrm{~mm} \& 6 \%$ taper) was used.

\subsubsection{Implementation of Taguchi DoE optimization}

In this study, three root canal preparation process parameters including root canal curvature angle, speed of endodontic file and type of endodontic file motion were selected as contributing factors. These process parameters were selected after consultation with the dental practitioners. A deign of experiments was used to study the interaction between contributing factors (input variables) and fatigue life of file (output variable). The full factorial DoE was performed, which helps to adjust the input to get optimized output. The orthogonal array L18 was selected consisting of 18 experimental runs. Three levels of root canal curvature angle, speed of endodontic file and two levels for type of endodontic file motion were considered.
During the preparation of root canal cavity endodontic file operates under either purely rotary or combined rotaryreciprocating motion, therefore two types of motions parameters were selected. The speeds of endodontic files were selected as per the manufacturer's catalog. The set of experimental runs shown in Table 6 corresponds to Taguchi Optimization Method.

The Taguchi method converts the experimental values to the signal-to-noise $(\mathrm{S} / \mathrm{N})$ ratio [13], the noise representing undesired values (Standard deviation) and the signal representing the desired values (Mean) for the output characteristics.

The optimization of root canal preparation parameters was done using Taguchi analysis for better fatigue life of the endodontic file. In the present study, three root canal preparation process parameters including root canal curvature angle, speed of endodontic file and type of endodontic file motion were selected as contributing factors. As per L-18 orthogonal array (shown in Tab. 7) experimentation was planned and executed. The experi- 
Table 6. Experimental design with contributing factors.

\begin{tabular}{llll}
\hline & & Root canal preparation parameters & \\
\cline { 2 - 4 } Levels & Motion type & Root canal angle & Speed of file \\
\hline 1 & & $23^{\circ}$ & 500 \\
2 & & $23^{\circ}$ & 600 \\
3 & Purely rotary & $23^{\circ}$ & 700 \\
4 & & $33^{\circ}$ & 500 \\
5 & & $33^{\circ}$ & 600 \\
6 & & $33^{\circ}$ & 700 \\
7 & & $43^{\circ}$ & 500 \\
8 & & $43^{\circ}$ & 600 \\
9 & & $43^{\circ}$ & 700 \\
10 & $23^{\circ}$ & 500 \\
11 & & $23^{\circ}$ & 600 \\
12 & $23^{\circ}$ & 700 \\
13 & & $33^{\circ}$ & 500 \\
14 & & $33^{\circ}$ & 600 \\
15 & $33^{\circ}$ & 700 \\
16 & & $43^{\circ}$ & 500 \\
18 & & $43^{\circ}$ & 600 \\
\hline
\end{tabular}

mental fatigue life values are then converted into an $\mathrm{S} / \mathrm{N}$ ratio and the higher the better is used to determine the output characteristics.

The endodontic file operates in the curved root canal cavity during the preparation process. Therefore, root canal curvature angle plays a vital role as it causes the stress state in file material. In another way speed of file and type of motion also causes stress state. As mentioned earlier Taguchi method was used for better fatigue life of the endodontic file. To get an exact idea about the role of these three parameters in fatigue failure of the file, the $\%$ contribution needs to be calculated.

\section{Result and discussion}

The present study successfully conducted a numerical simulation of the endodontic file and optimization of the endodontic file using Taguchi Design of Experiments (DoE). The study has used finite element analysis and cyclic fatigue test for assessment of fatigue life of endodontic files during the root canal preparation process when placed in $23^{\circ}, 33^{\circ}$ and $43^{\circ}$ root canal curvature angles and under purely rotary, combined reciprocating-rotary motions. Root canal curvature angles viz. $23^{\circ}, 33^{\circ}$ and $43^{\circ}$ were selected since for any patient the angle varies from $13^{\circ}$ to $45^{\circ}$. Therefore, to find cyclic fatigue resistance, three anles steps $23^{\circ}, 33^{\circ}$ and $43^{\circ}$ were selected. The Taguchi DoE optimization was used to find optimized root canal angle and speed of rotation giving maximum fatigue life of the file. Regression analysis and ANNOVA were used for statistical analysis of obtained results.

The FEA of the endodontic files indicated that Hyplex $\mathrm{CM}$ file works longer during the root canal preparation process under curved canals of $23^{\circ}, 33^{\circ}$ and $43^{\circ}$. Therefore, Hyplex CM endodontic file was for later study viz. optimization using Taguchi Design of Experiments (DoE). The optimized root canal preparation process parameters were estimated using the Taguchi method. The Taguchi DoE helped to find the optimized parameters which give maximum fatigue life. Each experimental run was conducted using X Smart Plus experimental set-up as shown in Figure 8. Regression analysis was performed to estimate the relationship between contributing and output variables to assess the closeness of experimentation with the developed model. The $\%$ contribution of parameters viz. root canal curvature angle, speed of endodontic file and type of endodontic file motion were decided using ANNOVA.

In the root canal preparation process, regression analysis was performed to estimate the relationship between contributing and output variables and to assess the closeness of experimentation with the developed model. In this study three root canal preparation process parameters viz. root canal curvature angle, speed of endodontic file and type of endodontic file motion were selected as contributing factors and fatigue life of endodontic file as output. The linear regression model is used to check if the data of fatigue life signify or not the output characteristic. 
Table 7. Experimental design using L-18 orthogonal array.

\begin{tabular}{|c|c|c|c|c|c|}
\hline \multirow[b]{2}{*}{$\begin{array}{l}\text { Experimental } \\
\text { Run }\end{array}$} & \multicolumn{3}{|c|}{ Orthogonal Array } & \multirow{2}{*}{$\begin{array}{l}\text { Experimental results } \\
\text { Fatigue life } \\
\text { of file }\end{array}$} & \multirow{2}{*}{ 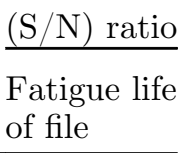 } \\
\hline & $\begin{array}{l}\text { Motion type } \\
\text { (MT) }\end{array}$ & $\begin{array}{l}\text { Curvature angle } \\
\text { of canal (CA) }\end{array}$ & $\begin{array}{l}\text { Speed of } \\
\text { file (SF) }\end{array}$ & & \\
\hline 1 & 1 & 1 & 1 & 6293 & -7.59772 \\
\hline 2 & 1 & 1 & 2 & 6122 & -7.57379 \\
\hline 3 & 1 & 1 & 3 & 5789 & -7.52521 \\
\hline 4 & 1 & 2 & 1 & 1629 & -6.42384 \\
\hline 5 & 1 & 2 & 2 & 1528 & -6.36825 \\
\hline 6 & 1 & 2 & 3 & 1388 & -6.28478 \\
\hline 7 & 1 & 3 & 1 & 1008 & -6.00692 \\
\hline 8 & 1 & 3 & 2 & 905 & -5.9133 \\
\hline 9 & 1 & 3 & 3 & 798 & -5.80401 \\
\hline 10 & 2 & 1 & 1 & 5605 & -7.49715 \\
\hline 11 & 2 & 1 & 2 & 5178 & -7.42832 \\
\hline 12 & 2 & 1 & 3 & 4771 & -7.35722 \\
\hline 13 & 2 & 2 & 1 & 1255 & -6.19729 \\
\hline 14 & 2 & 2 & 2 & 902 & -5.91041 \\
\hline 15 & 2 & 2 & 3 & 801 & -5.80727 \\
\hline 16 & 2 & 3 & 1 & 869 & -5.87804 \\
\hline 17 & 2 & 3 & 2 & 798 & -5.80401 \\
\hline 18 & 2 & 3 & 3 & 680 & -5.66502 \\
\hline
\end{tabular}

Table 8. Regression analysis.

Regression statistics

Multiple $R$

0.0754

$R$ Square

0.8533

Adjusted $R$ square

$-0.8565$

Standard error

2320.70

Observations

18

$\mathrm{R}$-square in the regression table indicates the effectiveness of the developed model. The value of $\mathrm{R}$-square if equals to 1 then it indicates the $100 \%$ effectiveness of model. In the present study $\mathrm{R}$-square is more than $80 \%$, which represents that model is effective to predict relationship between contributing factors (viz. root canal curvature angle, speed of endodontic file and type of endodontic file motion) and response (viz. life of endodontic file).

The ANNOVA was conducted for analyzing obtained data. Significance F and P-value in ANNOVA table, ideally, should be as small as possible. For example, in Table 9, the $P$-value is $0.36 \%$, regression model and intercept in Table 10 are $99.64 \%$ accurate. Significance $F$ is also very small, therefore, regression is good. The present ANNOVA analysis was conducted at $95 \%$ confidence interval. The obtained $P$ value is higher than the accepted confidence interval.

\section{Conclusion}

The present study concludes that the strain life analysis done using ANSYS showed that Hyplex CM file gives the highest fatigue life at $23^{\circ}, 33^{\circ}$ and $43^{\circ}$ root canal curvature angles. Taguchi DoE study performed, as per L-18 orthogonal array, showed that under purely rotary motion Hyplex $\mathrm{CM}$ file gives maximum fatigue life at $23^{\circ}$ root canal curvature angle and $500 \mathrm{rpm}$ speed of rotation. If file motion is combined rotary-reciprocating, life reduces drastically, since debries inside the root canal apply more resistance to the reciprocating motion of file than purely rotary. Concerning regression analysis done, $R$-square is $85.33 \%$, which represents that model is effective to predict the relationship between contributing factors (viz. root canal curvature angle, speed of endodontic file and type of endodontic file motion) and response (viz. life of endodontic file). On the other hand, ANNOVA showed that $P$-value and significance $F$ are very small, which represented that the regression model is effective.

\section{Future scope}

The fatigue failure of endodontic file may be affected by many parameters such as size, taper, cross sectional area, 
Table 9. Analysis of variance (regression and residual).

\begin{tabular}{llllll}
\hline ANNOVA & DF & SS & MS & F & Significance F \\
\hline Regression & 1 & 492885.33 & 492885.33 & 0.0915 & 0.007662 \\
Residual & 17 & 86170322.28 & 5385645.14 & & \\
Total & 18 & 86663207.61 & & & \\
\hline
\end{tabular}

Table 10. Analysis of variance (intercept and variable).

\begin{tabular}{lllllll}
\hline & Coefficients & Std. Error & t Stat & P-value & Lower 95\% & Upper 95\% \\
\hline Intercept & 3789.28 & 4056.62 & 0.9341 & 0.003641 & -4810.37 & 12388.92 \\
Speed of file & -2.0267 & 6.699 & -0.3025 & 0.007662 & -16.229 & 12.1752 \\
\hline
\end{tabular}

rigidity, flexibility, direction of rotation of files and forces acting on the rotary instruments during working. The same can be studied in future to cover more research insights.

\section{References}

1. R.R. Ghorpade, K. Sundaram, V. Hegde, Deformation studies of endodontic obturator tip using FEM approach, Int. J. Eng. Invent. 1, 19-26 (2012)

2. A. Ciftci, D.A. Vardarlı, I.S. Sonmez, Coronal micro-leakage of four endodontic temporary restorative materials: an in vitro study, Oral Surg. Oral Med. Oral Pathol. Oral Radiol. Endodontol. 108, 067-070 (2009)

3. S. Moradi, M. Lomee, M. Gharechahim, Comparison of fluid filtration and bacterial leakage techniques for evaluation of micro-leakage in endodontics, Dental Res. J. 12, 109-112 (2015)

4. S.E.D.M. Saber, M.M. Nagy, E. Schafer, Comparative evaluation of the shaping ability of Wave One, $\mathrm{R}$ eciproc and One Shape single-file systems in severely curved root canals of extracted teeth, Int. Endodontic J. 48, 109-114 (2015)

5. Y. Shen, H.M. Zhou, Y.F. Zheng, B. Peng, M. Haapasalo, Current challenges and concepts of the thermo-mechanical treatment of nickel-titanium instruments, J. Endodontics 39, 163-172 (2013)
6. A. Scattina, M. Alovisi, D.S. Paolino, D. Pasqualini, N. Scotti, G. Chiandussi, E. Berutti, Prediction of cyclic fatigue life of nickel-titanium rotary files by virtual modelling and finite elements analysis, J. Endodont. 41, 867-870 (2015)

7. D. Montalvao, Q. Shengwen, M. Freitas, A study on the influence of $\mathrm{Ni}-\mathrm{Ti} \mathrm{M}$-Wire in the flexural fatigue life of endodontic rotary files by using Finite Element Analysis, Mater. Sci. Eng. C 40, 172-179 (2014)

8. E. Pedulla, G.R.M. La Rosa, S. Boninelli, O.G. Rinaldi, E. Rapisarda, H.C. Kim, Influence of different angles of file access on cyclic fatigue resistance of Reciproc and Reciproc Blue instruments, J. Endodont. 44, 849-855 (2018)

9. A. Jamleh, A. Alfadley, K. Alfouzan, Vertical force induced with waveone and waveone gold systems during canal shaping, J. Endodont. 44, 412-415 (2018)

10. Y. Shen, C. Tra, A. Hieawy, Z. Wang, M. Haapasalo, Effect of torsional and fatigue preloading on HyFlex EDM files, J. Endodont. 44, 643-647 (2018)

11. T. Ozyurek, G. Uslu, U. Inan, A comparison of the cyclic fatigue resistance of used and new glide path files, J. Endodont. 43, 477-480 (2017)

12. O.A. Peters, Current challenges and concepts in the preparation of root canal systems: a review, J. Endodont. 30, 559-567 (2004)

13. V.K. Vankanti, V. Ganta, Optimization of process parameters in drilling of GFRP composite using Taguchi method, J. Mater. Res. Technol. 3, 35-41 (2014)

Cite this article as: Pravin R. Lokhande, Sachin S. Salunkhe, Sethuraman Balaguru, Numerical simulation and experimentation of endodontic file using Taguchi DoE, Int. J. Simul. Multidisci. Des. Optim. 12, 32 (2021) 